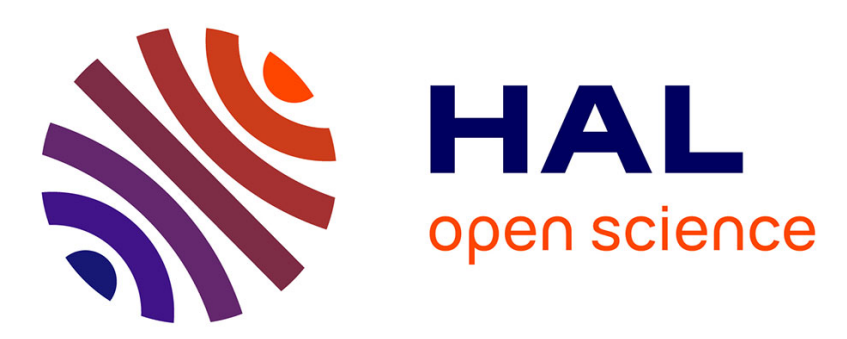

\title{
Embedding Naka-Rushton's equation in the geometric setting of Möbius transformations
}

Nicoletta Prencipe, Edoardo Provenzi

\section{To cite this version:}

Nicoletta Prencipe, Edoardo Provenzi. Embedding Naka-Rushton's equation in the geometric setting of Möbius transformations. ISPA - 12th Int'l Symposium on Image and Signal Processing and Analysis, Sep 2021, Zagreb, Croatia. hal-03276183

\section{HAL Id: hal-03276183 \\ https://hal.science/hal-03276183}

Submitted on 1 Jul 2021

HAL is a multi-disciplinary open access archive for the deposit and dissemination of scientific research documents, whether they are published or not. The documents may come from teaching and research institutions in France or abroad, or from public or private research centers.
L'archive ouverte pluridisciplinaire HAL, est destinée au dépôt et à la diffusion de documents scientifiques de niveau recherche, publiés ou non, émanant des établissements d'enseignement et de recherche français ou étrangers, des laboratoires publics ou privés. 


\title{
Embedding Naka-Rushton's equation in the geometric setting of Möbius transformations
}

\author{
Nicoletta Prencipe*1,2 and Edoardo Provenzi ${ }^{\dagger 1}$ \\ ${ }^{1}$ Université de Bordeaux, CNRS, Bordeaux INP, IMB, UMR 5251. F-33400, 351 \\ Cours de la Libération \\ ${ }^{2}$ Huawei France SASU
}

\begin{abstract}
The Naka-Rushton equation that models the transduction from electromagnetic energy carried by a photon to difference of potential of the membrane of a retinal photoreceptor has been used for two decades in tone mapping algorithms to compress the range of high dynamic range (HDR) images. Up to now, only its analytical properties of linear fractional transformation have been considered and exploited. In this paper, we recast the Naka-Rushton equation in the abstract setting of Möbius transformations, pointing out the hidden geometric properties of Naka-Rushton formula and discussing their pertinence to what a tone mapping algorithm is expected to comply.
\end{abstract}

\section{Introduction}

High Dynamic Range (HDR) images provide a way to store real-world radiance values by combining multiple photos of a static scene taken with different time exposures following the classical work of Debevec and Malik [7. However, since the real-world radiance can span up to 10 orders of magnitude and ordinary displays can only span up to two orders of magnitude, a further 'compression step', called 'Tone Mapping' (TM) is required to properly visualize the information stored in the HDR images.

Following Ward et al. 22, the large majority of tone mapping operators are devised to reproduce detail visibility and emulating as much as possible contrast and color sensation of the real-world scene. Ideally, a perfect model of the Human Visual System (HVS) would satisfy these requests, however the knowledge about human vision is still too vague to permit the construction of such a model, so that simplified and partial HVS descriptions are needed.

In this sense, it is quite remarkable that the sole use of the visual adaptation step already provides a remarkably good tone mapping. The visual adaptation phase, that will be recalled in section 2, occurs when photoreceptors (cones and rods) strongly compress the light range around an average (adaptation) value. This process can be described by the Naka-Rushton equation [15] which has been widely used as a global TM operator, for a thorough review see e.g. [20, 10, 11, [12, 17, 1].

The Naka-Rushton equation involves a fractional linear transformation that, up to our knowledge, has been analyzed so far only analytically, without taking into account its geometric properties. In this theoretical paper, we discuss these features from the point of view of Möbius transformations. The purpose of such an investigation is twofold: firstly, the knowledge of geometric properties underlying the Naka-Rushton formula may help scientists working on tone mapping to

*nicoletta.prencipe@math.u-bordeaux.fr

†edoardo.provenzi@math.u-bordeaux.fr 
more profoundly understand this formula and, possibly, to devise improvements for their algorithms. Secondly, as we will discuss in section 3 . Möbius transformations can be characterized as cross-ratio preserving maps, this turns out to be a crucial property in the framework of the very recent program of geometrization of colorimetry that can be found in papers [4, 18, 2, 16, 5, 6, 3].

\section{The Naka-Rushton equation and its use for tone mapping}

Let us begin this section by recalling how the retina responds to light stimuli. The range of radiances over which the HVS can operate is very large: from $10^{-6} \mathrm{~cd} / \mathrm{m}^{2}$ (scotopic limit) to $10^{6}$ $\mathrm{cd} / \mathrm{m}^{2}$ (glare limit) 23. The automatic process that allows the HVS to operate over such a huge range is called visual adaptation 21 .

However, the HVS cannot operate over this entire range simultaneously. Rather, it adapts to an average intensity and handles a smaller magnitude interval. There is no complete agreement in the literature about the precise value of this range, which can vary from two ([21] page 326) up to four orders of magnitude ([13] page 670).

Empirical experiments have shown that visual adaptation occurs mainly in the retina. The experiments to measure this behavior were performed using very simple, non-natural images: brief pulses of light with intensity $\mathcal{I}$ superimposed on a uniform background. When a photoreceptor absorbs $\mathcal{I}$, the electric potential of its membrane changes accordingly to the empirical law known in vision research literature as Naka-Rushton's equation [15, 21, 14]:

$$
r(\mathcal{I})=\frac{\mathcal{I}}{\mathcal{I}+\mathcal{I}_{s}},
$$

where $r(\mathcal{I})$ is the normalized response of the retina to $\mathcal{I}$ and $\mathcal{I}_{s}$ is the light level at which the photoreceptor response is half maximal, called semisaturation level and which is usually associated with the level of adaptation. Some authors report the formula writing the $\gamma$ power of $\mathcal{I}$ and $\mathcal{I}_{s}$, in this case the equation is called Michaelis-Menten's formula ([14 page 301).

The reason why this formula has received so much attention from the tone mapping community is because it permits to compress any given range of the light stimuli into the interval $[0,1]$ (since $\mathcal{I}$ and $\mathcal{I}_{s}$ are light intensity levels, hence they are both positive) in such a way that the details in dark areas are enhanced and the ones in the higher areas are compressed, see Fig. 2, which is coherent with the well-known Weber-Fechner's law 23.

Let us now see how Naka-Rushton's equation is used in the context of HDR imaging. We denote with $\Omega \subset \mathbb{R}^{2}$ the spatial domain of an RGB high dynamic range image $\vec{I}: \Omega \rightarrow(0,+\infty)^{3}$, $\Omega \ni x \mapsto\left(I_{R}(x), I_{G}(x), I_{B}(x)\right), I_{c}$ being the scalar chromatic component of $\vec{I}, c \in\{R, G, B\}$, where $x=\left(x_{1}, x_{2}\right) \in \Omega$ is the spatial position of an arbitrary pixel in the image. Generally, tone mapping algorithms operate on either the three RGB channels separately, or on the luminance value of $\vec{I}$, calculated in one of the many possible ways available in literature [20. In any case, it is clear that only a scalar-valued function is considered, let us denote it simply with $\lambda: \Omega \rightarrow(0,+\infty)$.

Since the HDR image represents the radiance map of a scene, it is natural to associate $\lambda$ with $\mathcal{I}$ and to identify the semisaturation level $\mathcal{I}_{S}$ with the average value of $\lambda$, denoted by $\mu$. In the literature there is no agreement about how $\mu$ has to be computed, i.e. via arithmetic average $\mu_{a}$, geometric average $\mu_{g}$, median $\mu_{\text {med }}$, or combinations of them 20, 10. For these reasons, and since our paper is meant to be merely theoretical, we will leave the formal expression of $\mu$ unspecified. In section 3 we will see that the parameter $\mu$ has a particular geometrical meaning in the decomposition of $r$ as a Möbius transformation.

With the notation just introduced, the expression of the Naka-Rushton equation for tone mapping of HDR images is the following:

$$
r(\lambda(x))=\frac{\lambda(x)}{\lambda(x)+\mu}, \quad \forall x \in \Omega .
$$

In the so-called local tone mapping algorithms, both the pixel position $x$ and the value of $\lambda(x)$ influence the tone mapping operation, however, for the sake of simplicity, in this paper we will 
only deal with a global tone mapping, in which two generic pixels $x, y \in \Omega$ such that $\lambda(x)=\lambda(y)$ will be tone-mapped in exactly the same value. Thanks to this assumption, formula (2) can be simplified as follows:

$$
r(\lambda)=\frac{\lambda}{\lambda+\mu}, \quad \forall \lambda \in\left[\lambda_{\min }, \lambda_{\max }\right] \subset(0,+\infty),
$$

with obvious meaning of the symbols $\lambda_{\min }$ and $\lambda_{\max }$ and $r(\mu)=1 / 2$. The graphical behavior of $r(\lambda)$ is depicted in Fig. 2. The non-linearity of $r(\lambda)$ is essential, in fact, due to the vast dynamic range of HDR images, a linear tone mapping performed via the formula:

$$
\lambda \mapsto \frac{\lambda-\lambda_{\min }}{\lambda_{\max }-\lambda_{\min }} \in[0,1],
$$

eventually multiplied by 255 to restore the usual 8 bit dynamic range, would set to black all pixels in the image whose $\lambda$ value is two orders of magnitude smaller than $\lambda_{\max }$, as clearly shown in Fig. 1.

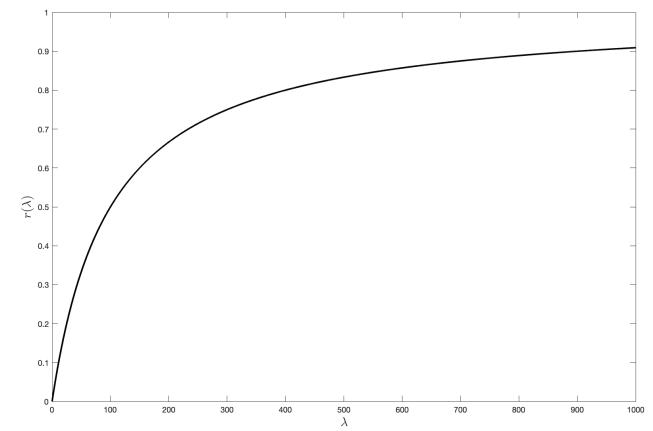

Figure 1: Graph of $r(\lambda)$, with $\lambda_{\min }=0.1, \lambda_{\max }=10^{3}$ and $\mu=100$.
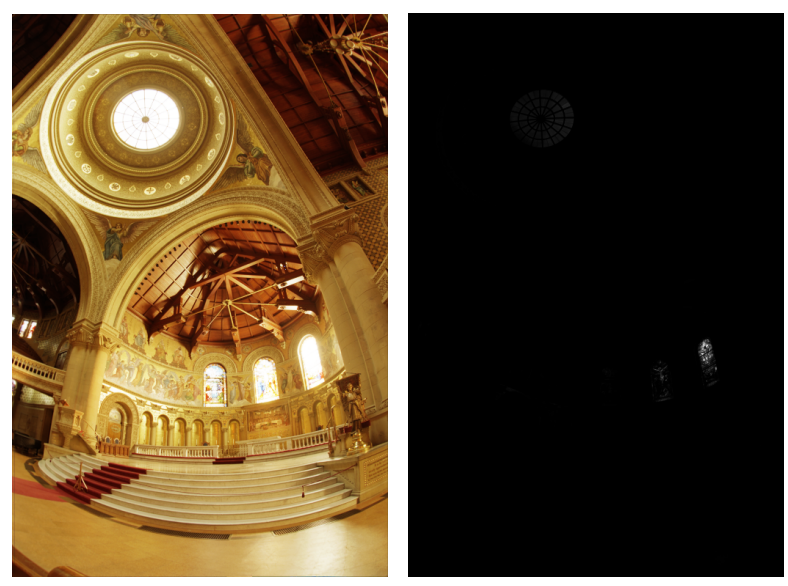

Figure 2: Left: one image of the famous Memorial church sequence. Right: result of the linear tone mapping of eq. (4), where almost all the pixels are set to black apart from those belonging to the brightest areas of the image. 


\section{Analysis of Naka-Rushton's formula as a Möbius trans- formation}

The non-linearity of the Naka-Rushton transformation and the possibility to quite easily control the global brightness of the resulting image by modifying the value of $\mu$ are the two most important features of Naka-Rushton based tone mapping that established its successful and widespread use.

Alongside the analytical formula (3) and the graphical depiction of Fig. 2, it is possible to analyze the Naka-Rushton transformation from a geometric point of view that happens to give finer information about its behavior. In fact, as we are going to prove in this section, eq. (3) can be interpreted as the composition of a non-linear map followed by an affine one.

In order to prove this, we must first introduce the concepts of reflection, inversion and Möbius transformation.

\subsection{Möbius transformations}

The main reference for this section is the book [19.

One way to define the group of Möbius transformations on $\mathbb{R}^{n}$, denoted by $\mathcal{M}\left(\mathbb{R}^{n}\right)$ is as the subgroup of $\operatorname{Aut}\left(\mathbb{R}^{n}\right)=\left\{f: \mathbb{R}^{n} \rightarrow \mathbb{R}^{n}, f\right.$ bijective $\}$ generated by reflections w.r.t. hyperplanes and inversions w.r.t. hyperspheres.

The concepts of hyperplane and hypersphere are the generalizations to dimension $n$ of the concepts of plane and sphere in dimension 3 or straight line and circle in dimension 2 . It is important to stress that both hyperplanes and hyperspheres are submanifolds of dimension $n-1$ embedded in a space of dimension $n$.

Def. 3.1 Given $a \in \mathbb{R}^{n},\|a\|=1$, and $t \geq 0$, the hyperplane in $\mathbb{R}^{n}$ associated to $a$ and $t$ is the set

$$
P_{a, t}:=\left\{x \in \mathbb{R}^{n},\langle x, a\rangle=t\right\} .
$$

Note that $a$ is the normal vector to $P_{a, t}$, and $t$ is the distance between $P_{a, t}$ and 0 .

Def. 3.2 A reflection in $\mathbb{R}^{n}$ w.r.t. the hyperplane $P_{a, t}$ is the affine function:

$$
\begin{aligned}
\rho_{a, t}: \mathbb{R}^{n} & \longrightarrow \mathbb{R}^{n} \\
x & \mapsto \rho_{a, t}(x):=x+2(t-\langle x, a\rangle) a .
\end{aligned}
$$

We denoted with $\langle\cdot, \cdot\rangle$ the Euclidean scalar product.

Geometrically, the reflection $\rho_{a, t}$ takes any point $x \in \mathbb{R}^{n}$ at a distance $d$ from $P_{a, t}$ to a point $\rho_{a, t}(x)$ which lies specularly on the other side of $P_{a, t}$ at the same distance $d$.

Proposition 3.1 Every reflection $\rho_{a, t}$ satisfies the following properties for all $x, y \in \mathbb{R}^{n}$ :

1. $\rho_{a, t}(x)=x$ if and only if $x \in P_{a, t}$;

2. $\rho_{a, t}^{2}(x)=x$, i.e. $\rho_{a, t}$ is an involution, and so $\rho_{a, t}^{2}=\operatorname{id}_{\mathbb{R}^{n}}$, i.e. $\rho_{a, t}$ is a bijection with $\rho_{a, t}^{-1}=\rho_{a, t}$;

3. $\rho_{a, t}$ is a Euclidean isometry: $\left\|\rho_{a, t}(x)-\rho_{a, t}(y)\right\|=\|x-y\|$.

Def. 3.3 Given $c \in \mathbb{R}^{n}$ and $d>0$, the hypersphere, of dimension $n-1$, in $\mathbb{R}^{n}$ centered in $c$ of radius $d$ is the set of all the points having distance $d$ from the center $c$ :

$$
S_{c, d}:=\left\{x \in \mathbb{R}^{n}:\|x-c\|=d\right\} .
$$


The main difference between reflections and inversions is that the hypersurface w.r.t. the inversion is performed is not a hyperplane but a hypersphere. While a hyperplane extends towards the infinite, a sphere is bounded, this fact implies that it is impossible to continuously fill the whole outer space to the spherical surface simply by reflecting its interior points w.r.t. the tangent hyperplane to the sphere at a point, a different, non-linear, geometrical operation is needed.

This operation consists in mapping any point $x$ inside the sphere to the unique point $\sigma(x)$ outside the sphere characterized by the following two properties: firstly, $\sigma(x)$ lies on the same line joining $x$ with the center of the sphere; secondly, the norm of $\sigma(x)$ is inverted w.r.t. that of $x$.

More precisely we have the following definition:

Def. 3.4 Let $c \in \mathbb{R}^{n}$ and $d>0$, then the inversion in $\mathbb{R}^{n}$ w.r.t. the hypersphere $S_{c, d}$ is the non-linear function

$$
\begin{aligned}
& \sigma_{c, d}: \mathbb{R}^{n} \backslash\{c\} \quad \longrightarrow \quad \mathbb{R}^{n} \backslash\{c\} \\
& x \quad \mapsto \quad \sigma_{c, d}(x):=c+\frac{d^{2}}{\|x-c\|^{2}}(x-c) .
\end{aligned}
$$

$\sigma_{a, d}(x)$ is said to be the inverse of $x$ w.r.t. to the sphere $S_{c, d}$.

Proposition 3.2 Every inversion $\sigma_{c, d}$ w.r.t. a hypersphere $S_{c, d}$ satisfies the following properties for all $x, y \in \mathbb{R}^{n} \backslash\{c\}$ :

1. $\sigma_{c, d}(x)=x$ if and only if $x \in S_{c, d}$;

2. $\sigma_{c, d}^{2}(x)=x$, i.e. $\sigma_{c, d}$ is an involution, and so $\sigma_{c, d}$ is invertible with $\sigma_{c, d}{ }^{-1}=\sigma_{c, d}$;

3. $\left\|\sigma_{c, d}(x)-\sigma_{c, d}(y)\right\|=\frac{d^{2}}{\|x-c\|\|y-c\| \|}\|x-y\|$.

Every circular inversion $\sigma_{c, d}$ maps the exterior part of the hypersphere $\bar{B}_{c, d}^{c}=\left\{x \in \mathbb{R}^{n}\right.$ : $\|x-c\|>d\}$ into the inner part $B_{c, d}=\left\{x \in \mathbb{R}^{n}:\|x-c\|<d\right\}$ and viceversa, i.e. $\sigma\left(\bar{B}_{c, d}^{c}\right)=B_{c, d}$ and $\sigma\left(B_{c, d}\right)=\bar{B}_{c, d}^{c}$ while the points of $S_{c, d}$ stay fixed, i.e. $\sigma\left(S_{c, d}\right)=S_{c, d}$.

Both reflections w.r.t. hyperplanes and inversions w.r.t. hyperspheres are, essentially, onedimensional operations, in the sense that all the points belonging to the same straight line orthogonal to the hyperplane involved in a reflection are left on this straight line; in the same way, all the points belonging to the straight line passing through the origin of the sphere involved in an inversion are left on that line.

It is possible to extend reflections and inversions to $\widehat{\mathbb{R}}^{n}:=\mathbb{R}^{n} \cup\{\infty\}$. It is sufficient to define the image of $\infty$.

Def. 3.5 Let $\rho_{a, t}$ be a reflection and $\sigma_{c, d}$ an inversion in $\mathbb{R}^{n}$. The extension of $\rho_{a, t}$ in $\infty$ and of $\sigma_{c, d}$ in $\infty$ and $c$ are defined as follows:

$$
\rho_{a, t}(\infty):=\infty \quad \text { and } \quad\left\{\begin{array}{l}
\sigma_{c, d}(\infty):=c \\
\sigma_{c, d}(c):=\infty
\end{array} .\right.
$$

Möbius transformations arise from the combinations of inversions and reflections of $\widehat{\mathbb{R}}^{n}$, one of the main interest in combining them is that, when they are fused together, they form a group. Notice that this is not a trivial statement because neither the set of reflections nor the set inversions form a group: we do not have a identity element or any stability.

Def. 3.6 A Möbius transformation $\phi: \widehat{\mathbb{R}}^{n} \rightarrow \widehat{\mathbb{R}}^{n}$ is a finite composition of reflections w.r.t. a hyperplane and inversions w.r.t. a sphere in $\widehat{\mathbb{R}}^{n}$. The group of Möbius transformations is: $\mathcal{M}\left(\widehat{\mathbb{R}}^{n}\right)=\left\{\phi=\mu_{1} \circ \cdots \circ \mu_{m}: m \in \mathbb{N}, \mu_{i}\right.$ reflections or inversions of $\left.\widehat{\mathbb{R}}^{n}, i \in\{1, \ldots, m\}\right\}$.

Another equivalent characterization of Möbius transformations is based on the concept of cross-ratio. 
Def. 3.7 Let $u, v, x, y \in \widehat{\mathbb{R}}^{n}$ such that $u \neq y, v \neq x$. The cross-ratio of $(u, v, x, y)$ is given by:

$$
[u, v, x, y]=\frac{(u-x)(v-y)}{(u-y)(v-x)}
$$

$\mathcal{M}\left(\widehat{\mathbb{R}}^{n}\right)$ can be characterized as the set of all the transformations preserving the cross-ratio, i.e. $\phi \in \mathcal{M}\left(\widehat{\mathbb{R}}^{n}\right)$ if and only if for all $u, v, x, y \in \widehat{\mathbb{R}}^{n}$ we have that $[u, v, x, y]=[\phi(u), \phi(v), \phi(x), \phi(y)]$.

Theorem 3.1 Let $\phi \in \mathcal{M}\left(\widehat{\mathbb{R}}^{n}\right)$. Then:

1. $\phi(\infty)=\infty$ if and only if $\phi$ is a similarity of $\widehat{\mathbb{R}}^{n}$;

2. if $\phi(\infty) \neq \infty$, then, there exist:

- a unique hypersphere $\Sigma$ in $\mathbb{R}^{n}$ on which $\phi$ acts as a Euclidean isometry, i.e. for all $x, y \in \Sigma,\|\phi(x)-\phi(y)\|=\|x-y\|$;

- a unique inversion $\sigma$ w.r.t. $\Sigma$ and a unique Euclidean isometry $\psi$ such that $\phi$ can be decomposed as follows $\phi=\psi \circ \sigma$.

\subsection{Naka-Rushton's formula as a Möbius transformation}

Let us consider the real interval $\Lambda:=\left[\lambda_{\min }, \lambda_{\max }\right]$. The intensity levels of a HDR image belong to $\Lambda$, i.e. $\lambda(\Omega) \subset \Lambda$. We want to use a parsimonious combination of simple Möbius transformations, i.e. inversions and reflections, to obtain a non-linear compression of the interval $\Lambda$, hence at least one inversion is needed.

We will see that the search of the simplest monodimensional Möbius transformation $r$ such that:

1. $r$ is non-linear;

2. $\quad r$ is compressive $\rrbracket^{1}$ on $\Lambda$;

3. $r$ is monotonically increasing on $\Lambda$;

leads naturally to Naka-Rushton's transformation.

In the following we are going to use elements of $\mathcal{M}(\mathbb{R})$, hence $n=1$. Since the intensity is always positive, we can consider their restriction to $(0,+\infty)$. Notice that when $n=1$ a hyperplane is just a point $a \in \mathbb{R}$, while a hypersphere is the set of two points having the same distance $d$ from the center $c, S_{c, d}=\{c-d, c+d\}$.

In dimension 1 reflections and inversions have the following easier expressions:

$$
\rho_{a}(x)=a-x \quad \text { and } \quad \sigma_{c, d}(x)=c+\frac{d^{2}}{x-c},
$$

with $a, c \in \mathbb{R}$ and $d>0$.

Recalling theorem 3.1 a generic $r \in \mathcal{M}(\mathbb{R})$ is either a similarity or not, and it can be decomposed as $r=\psi \circ \sigma$, where $\psi$ is an isometry of $\mathbb{R}$ and $\sigma$ is a (unique) circular inversion. Since we are looking for a non-linear transformation and similarities act linearly, we can exclude the first option.

We want $r$ to be compressive on $\Lambda$. Since the component $\psi$ is an isometry ${ }^{2}, \sigma$ must be compressive on $\Lambda$.

Let us consider the simplest case of a circular inversion w.r.t. a sphere centered in 0 of radius $1, S_{0,1}=\{-1,1\}$. The circular inversion w.r.t. this sphere is given by $\sigma_{0,1}(x)=\frac{x}{\|x\|^{2}}$, and, since we are in dimension $1, \sigma_{0,1}(x)=\frac{1}{x}$.

\footnotetext{
${ }^{1}$ For all $\lambda_{1}, \lambda_{2} \in \Lambda$ we have that $\left|r\left(\lambda_{1}\right)-r\left(\lambda_{2}\right)\right|<\left|\lambda_{1}-\lambda_{2}\right|$.

${ }^{2}$ For all $\lambda_{1}, \lambda_{2} \in \Lambda$ we have that $\left|\psi\left(\lambda_{1}\right)-\psi\left(\lambda_{2}\right)\right|=\left|\lambda_{1}-\lambda_{2}\right|$.
} 
Notice that, as an inversion, $\sigma_{0,1}$ maps the inner part of $S_{0,1}$ into the external part and viceversa, i.e. $\sigma_{0,1}((0,1))=(1,+\infty)$ and $\sigma_{0,1}((1,+\infty))=(0,1)$. Since $\sigma_{0,1}(1)=1$ we can also say that $\sigma_{0,1}([1,+\infty))=(0,1]$. In other words, this last equality means that the infinite half line $(1,+\infty)$ is mapped into the bounded segment $(0,1)$.

This means that, if the interval $\Lambda$, which can have arbitrarily large length $l=\lambda_{\max }-\lambda_{\min }$, is contained in the half line $[1,+\infty)$, then $\sigma_{0,1}(\Lambda)$ will be compressed, not linearly, in a segment contained in $(0,1]$. To ensure that $\Lambda \subset[1,+\infty)$ we preliminary apply the translation $\tau_{1}(x)=x+1$, hence $\tau_{1}(\Lambda)=\left[\lambda_{\min }+1, \lambda_{\max }+1\right]$.

Up to now, we are applying on $\Lambda$ the transformation $\sigma_{0,1} \circ \tau_{1}(x)=\frac{1}{x+1}$, see Fig. 3. Although this function is non-linear and compressive on $\Lambda$, it does not respect the order on $\Lambda$, i.e. it is not monotonically increasing. To recover the correct order on the compressed interval we need to apply the reflection w.r.t. the point $\frac{1}{2}$, i.e. $\rho_{1 / 2}(x)=1-x$. Note that, in particular, $\rho_{1 / 2}$ reverses the segment $[0,1]$, indeed it swaps the segment's extremes: $\rho_{1 / 2}(0)=1$ and $\rho_{1 / 2}(1)=0$. As a reflection $\rho_{1 / 2}$ is an isometry thanks to proposition 3.1 .

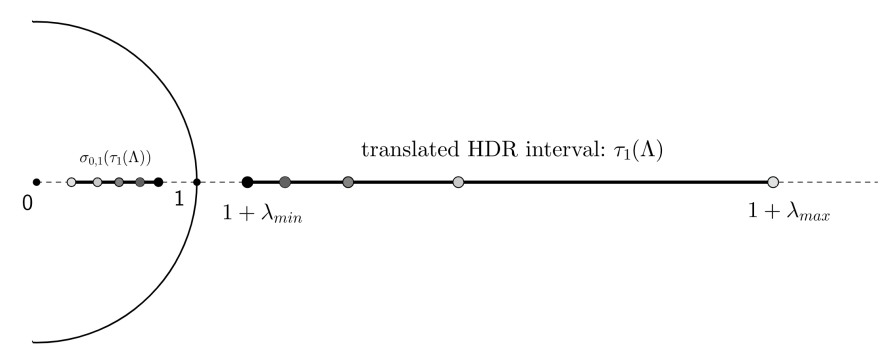

Figure 3: Depiction of the action of the transformation $\rho_{1 / 2} \circ \sigma_{0,1}$ on the interval $\Lambda$. Notice that it is more compressive on high intensity levels than on the lower ones.

Let us call $r^{\prime}=\rho_{1 / 2} \circ \sigma_{0,1}$ and $r=r^{\prime} \circ \tau_{1}$. In this case, without taking into account the preliminary translation $\tau_{1}$, i.e. considering $r^{\prime}$ instead of $r, r^{\prime}$ is decomposed as stated in theorem 3.1 i.e. $r^{\prime}=\psi \circ \sigma$, with $\psi=\rho_{1 / 2}$ and $\sigma=\sigma_{0,1}$. Moreover $r$ has the following explicit expression:

$$
r(x)=\rho_{1 / 2} \circ \sigma_{0,1} \circ \tau_{1}(x)=\rho_{1 / 2}\left(\frac{1}{x+1}\right)=\frac{x}{x+1} .
$$

Notice that this simple reasoning leads us to a Naka-Rushton transformation with $\mu=1$.

Let us follow the same reasoning, but starting from a more generic circular inversion $\sigma_{0, \mu}$ w.r.t. a sphere centered in 0 of radius $\mu, S_{0, \mu}=\{-\mu, \mu\}$.

To ensure that $\Lambda$ is placed outside the sphere $S_{0, \mu}$, i.e. $\Lambda \subset[\mu,+\infty)$, before the inversion $\sigma_{0, \mu}$, we preliminary apply the translation $\tau_{\mu}(x)=x+\mu$.

Up to now, we are considering the transformation $\sigma_{0, \mu} \circ \tau_{\mu}$. We know that $\sigma_{0, \mu}(x)=\frac{\mu^{2} x}{|x|^{2}}$, but, since we are in dimension $1, \sigma_{0, \mu}(x)=\frac{\mu^{2}}{x}$. Hence $\sigma_{0, \mu} \circ \tau_{\mu}(x)=\frac{\mu^{2}}{x+\mu}$. Now $\sigma_{0, \mu} \circ \tau_{\mu}(\Lambda)$ is contained in the segment $[0, \mu]$. To recover the correct order of the intensity levels we need to apply the reflection $\rho_{\mu / 2}(x)=\mu-x$, which reverses the segment $t^{3}[0, \mu]$. At this point the image of $\Lambda$ is contained in the segment $[0, \mu]$, we need to rescale it applying a dilation of factor $\frac{1}{\mu}, \delta_{1 / \mu}(x)=\frac{1}{\mu} x$, which maps $[0, \mu]$ into $[0,1]$. In this way the image of $\Lambda$ is contained in $[0,1]$. Let us call $r$ the composition of all the Möbius transformations introduced up to now:

$$
r(x)=\delta_{1 / \mu} \circ \rho_{\mu / 2} \circ \sigma_{0, \mu} \circ \tau_{\mu}(x)=\frac{x}{x+\mu} .
$$

\footnotetext{
${ }^{3}$ Indeed it switches the segment's extremes, i.e. $\rho_{\mu / 2}(0)=\mu$ and $\rho_{\mu / 2}(\mu)=0$.
} 
The Möbius transformation $r$ that we have obtained is a Naka-Rushton function and the parameter $\mu$ represents the radius of the sphere w.r.t. which we are performing a circular inversion.

Let us call $r^{\prime}=\rho_{\mu / 2} \circ \sigma_{0, \mu}$, hence $r=\delta_{1 / \mu} \circ r^{\prime} \circ \tau_{\mu}$. If we do not consider the preliminary translation $\tau_{\mu}$ and the dilation $\delta_{1 / \mu}$ that we apply a posteriori to recover the range $[0,1]$, i.e. we consider $r^{\prime}$ instead of $r$, then $r^{\prime}$ agrees with the minimal decomposition stated in theorem 3.1 as $r^{\prime}=\psi \circ \sigma$, with $\psi=\rho_{\mu / 2}$ and $\sigma=\sigma_{0, \mu}$.

One might also think to decompose $r=\frac{x}{x+\mu}$ according to theorem 3.1. After straightforward computations it is easy to obtain that $r=\psi \circ \sigma$ with $\psi(x)=\rho_{\frac{1-\mu}{2}}(x)=1-\mu-x$ and $\sigma(x)=$ $\sigma_{-\mu, \sqrt{\mu}}(x)=-\mu+\frac{\mu}{x+\mu}$.

Notice that also in this decomposition the parameter $\mu$ is related to the radius of the sphere w.r.t. which we are performing an inversion.

\section{Discussion}

We have explicitly identified the non-linear inversion and the affine reflection that make up NakaRushton's map as a Möbius transformation. An inversion encodes the non-linear part of the mapping, mapping very dark and bright values of $\lambda$ in the range $0-255$ with the reversed order between them. For this reason, the composition with a reflection is needed to re-establish the correct intensity order.

The fact that reflections are affine maps is particularly important for this step, in fact they do not dramatically modify the image intensities obtained as output of the inversion step, which is wise because the range has been correctly shrunk and we want to preserve it like it is.

We consider that pointing out this geometric information about tone mapping operator based on Naka-Rushton's formula may give interesting information for future developments of this kind of techniques. In particular, considering the prominent role of Möbius transformations in hyperbolic geometry and the importance played by hyperbolic structures in the program of geometrization of colorimetry developed in the papers $4,18,2,16,5,6,3$.

Möbius transformations encode many desirable colorimetric properties, e.g. they happen to coincide with conformal maps, i.e. they preserve the angle between vectors, but they can also be identified with the transformations that preserve the cross-ratio (see e.g. [19] for more details), which is related to Weber ratio, and thus to human vision, see e.g. [8, 9].

In this paper we have considered only 1-dimensional Möbius transformations that can either be applied on the luminance channel of an RGB image, or to each separate chromatic channel, or, again, any other 1-dimensional decomposition of the image in a given color space. It would be interesting to study also the application of 3-dimensional Möbius transformations, i.e. elements of $\mathcal{M}\left(\mathbb{R}^{3}\right)$, on the whole image content.

\section{Acknowledgments}

This study has been carried out with financial support of Huawei Technologies France SASU.

\section{References}

[1] Nikola Banic and Sven Loncaric. Flash and Storm: Fast and highly practical tone mapping based on Naka-Rushton equation. In VISIGRAPP (4: VISAPP), pages 47-53, 2018. https: //doi.org/10.5220/0006621600470053.

[2] M. Berthier. Geometry of color perception. Part 2: perceived colors from real quantum states and Hering's rebit. The Journal of Mathematical Neuroscience, 10(1):1-25, 2020.

[3] M. Berthier, V. Garcin, N. Prencipe, and E. Provenzi. The relativity of color perception. Submitted to Journal of Mathematical Psychology, preprint available at https: //hal. archives-ouvertes. fr/hal-02546380v2/document. 
[4] M. Berthier and E. Provenzi. When geometry meets psycho-physics and quantum mechanics: Modern perspectives on the space of perceived colors. In GSI 2019, volume 11712 of Lecture Notes in Computer Science, pages 621-630. Springer Berlin-Heidelberg, 2019. https://doi. org/10.1007/978-3-030-26980-764.

[5] M. Berthier and E. Provenzi. From riemannian trichromacy to quantum color opponency via hyperbolicity. Journal of Mathematical Imaging and Vision, 10(1):1-25, 2021. https: //doi.org/10.1007/s10851-021-01023-5.

[6] Michel Berthier and Edoardo Provenzi. The quantum nature of color perception: Uncertainty relations for chromatic opposition. Journal of Imaging, 7(2):40, 2021. https://doi.org/10. 3390/jimaging7020040.

[7] P. Debevec and J. Malik. Recovering high dynamic range radiance maps from photographs. In Proc. of the 24th annual conf. on Computer graphics, pages 369-378, 1997. https://doi. org/10.1145/258734.258884

[8] Jan Drösler. Color similarity represented as a metric of color space. In Contributions to mathematical psychology, psychometrics, and methodology, pages 19-37. Springer, 1994. https://doi.org/10.1007/978-1-4612-4308-32.

[9] Jan Drösler. The invariances of weber's and other laws as determinants of psychophysical structures. Geometric representations of perceptual phenomena, 1995. https://doi.org/10. 4324/9780203773949.

[10] S. Ferradans, M. Bertalmío, E. Provenzi, and V. Caselles. An analysis of visual adaptation and contrast perception for tone mapping. IEEE Transactions on Pattern Analysis and Machine Intelligence, 33(10):2002-2012, 2011. https://doi.org/10.1109/TPAMI.2011.46.

[11] S. Ferradans, M. Bertalmío, E. Provenzi, and V. Caselles. Generation of hdr images in non-static conditions based on gradient fusion. In Proceedings of the VISAPP Conference, February 24-26, Rome, Italy, pages 31-37. SciTePress, 2012. https://doi.org/10.5220/ 0003840700310037 .

[12] S. Ferradans, R. Palma-Amestoy, and E. Provenzi. An algorithmic analysis of variational models for perceptual local contrast enhancement. Image Processing On Line, 5(10):219-233, 2015. https://doi.org/10.5201/ipol.2015.131.

[13] J. Keener and J. Sneyd. Mathematical Physiology. Springer, 2008. https://doi.org/10. 1016/0278-4327(84)90011-7.

[14] H-C. Lee. Introduction to Color Imaging Science. Cambridge University Press, 2005.

[15] K.I. Naka and W.A.H. Rushton. S-potentials from luminosity units in the retina of fish (cyprinidae). Journal of Physiology, 185:587-599, 1966. https://doi.org/10.1113/ jphysiol.1966.sp008003.

[16] N. Prencipe, V. Garcin, and E. Provenzi. Origins of hyperbolicity in color perception. Journal of Imaging, 42(6):1-19, 2020. https://doi.org/10.3390/jimaging6060042.

[17] Edoardo Provenzi. Computational Color Science: Variational Retinex-like Methods. John Wiley \& Sons, 2017. https://doi.org/10.1002/9781119407416.

[18] Edoardo Provenzi. Geometry of color perception. Part 1: Structures and metrics of a homogeneous color space. The Journal of Mathematical Neuroscience, 10(1):1-19, 2020. https://doi.org/10.1186/s13408-020-00084-x.

[19] J.G. Ratcliffe. Foundations of hyperbolic manifolds, volume 149. Springer. https://doi. org/10.1007/978-0-387-47322-2. 
[20] E. Reinhard, G. Ward, S. Pattanaik, and P. Debevec. High Dynamic Range Imaging, Acquisition, Display, And Image-Based Lighting. Morgan Kaufmann Ed., 2005. https: //doi.org/10.1016/B978-0-12-585263-0.X5000-3

[21] R. Shapley and C. Enroth-Cugell. Visual adaptation and retinal gain controls, volume 3, chapter 9, pages 263-346. Progress in Retinal Research, 1984. https://doi.org/10.1016/ 0278-4327(84) 90011-7.

[22] G. Ward, H. Rushmeier, and C. Piatko. A visibility matching tone reproduction operator for high dynamic range scenes. IEEE Transactions on Visualization and Computer Graphics, 3:291-306, 1997. https://doi.org/10.1109/2945.646233.

[23] G. Wyszecky and W. S. Stiles. Color science: Concepts and methods, quantitative data and formulas. John Wiley 83 Sons. John Wiley \& Sons, 1982. https://doi.org/10.1002/col. 5080080421 . 